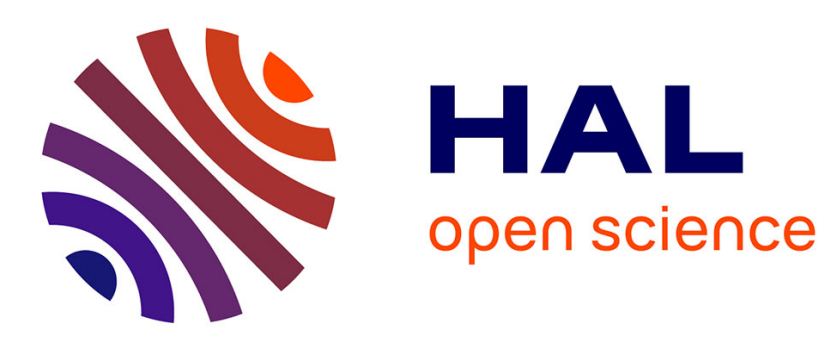

\title{
Non abelian 2-cohomology of semi-simple groups over rational varieties
}

Bénaouda Djamai

\section{To cite this version:}

Bénaouda Djamai. Non abelian 2-cohomology of semi-simple groups over rational varieties. International Journal of Algebra and Computation, 2016, 10, pp.549 - 557. 10.12988/ija.2016.61275 . hal-01768666

\section{HAL Id: hal-01768666 https://hal.science/hal-01768666}

Submitted on 19 Apr 2018

HAL is a multi-disciplinary open access archive for the deposit and dissemination of scientific research documents, whether they are published or not. The documents may come from teaching and research institutions in France or abroad, or from public or private research centers.
L'archive ouverte pluridisciplinaire HAL, est destinée au dépôt et à la diffusion de documents scientifiques de niveau recherche, publiés ou non, émanant des établissements d'enseignement et de recherche français ou étrangers, des laboratoires publics ou privés. 


\title{
Non abelian 2-cohomology of semi-simple groups over rational varieties
}

\author{
Bénaouda DJAMAI \\ UFR de Mathématiques \\ Université de Lille 1 \\ Sciences et Technologies \\ F-59665 Villeneuve d'Ascq Cedex France \\ In memory of Jean Claude DOUAI
}

\begin{abstract}
The aim of this paper is to study non abelian 2-cohomology of semisimple groups over rational varieties. The essential invariant is the $H^{1}(k, \operatorname{Pic}(\bar{X}))$, where $X$ is a rational variety over a $p$-adic field, or a number field, or more generally, a good field $k$ of cohomological dimension $\leqslant 2$. In particular, if $o b(X)=0(o b(X)$ : elementary obstruction $)$, we show that every class in $H^{2}(X, \mathcal{L})$, where $\mathcal{L}$ is an $X$-lien locally represented by a semi-simple simply connected group $\widetilde{G}$, becomes neutral by the base change $\mathcal{T}_{c}^{\prime} \rightarrow X^{\prime} \rightarrow X$, where $X^{\prime}$ is the Dynkin scheme of the associated $\widetilde{G}$ form $\widetilde{G}_{\mathcal{L}}$ to $\mathcal{L}$, and $\mathcal{T}_{c}^{\prime}$ a universal $X^{\prime}$-torsor compactification. This result confirms the fact that universal torsors compactifications have more simpler arithmetic.
\end{abstract}

\section{Subject Classification: 14F20}

Keywords: Rational varieties, Universal torsors, Elementary obstruction, Brauer group, Gerbs.

\section{On lower dimensions exact sequence}

Let $k$ be a perfect field and $X$ a proper, smooth, and geometrically integral $k$-variety which is $\bar{k}$-rational (we say also rational). From Leray spectral sequence:

$$
H^{p}\left(k, H^{q}\left(\bar{X}, G_{m}\right) \Rightarrow H^{p+q}\left(X, G_{m}\right)\right.
$$

we get the following exact sequence in lower dimensions:

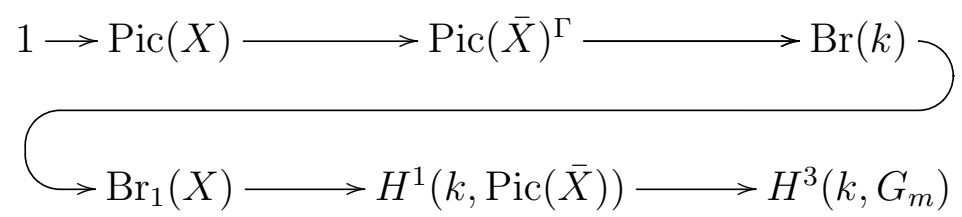

where $\operatorname{Br}_{1}(X)=\operatorname{Br}(X)^{t r}=\operatorname{Ker}\left\{\operatorname{Br}(X) \longrightarrow \operatorname{Br}\left(X \otimes_{k} \bar{k}\right)=\operatorname{Br}(\bar{X})\right\}$ 
Example 1.1 (cf. Prop. 1.5 of [1]): Let $C$ be a smooth conic defined over $k$ without $k$-rational point. Exact sequence (1) becomes :

$$
0 \longrightarrow \mathbb{Z} / 2 \longrightarrow \operatorname{Br} k \longrightarrow \operatorname{Br}(C) \longrightarrow 0
$$

where the generator of $\mathbb{Z} / 2$ maps to the quaternions algebra class associated to $C$.

Indeed, $\operatorname{Pic}(\bar{C})=\mathbb{Z}$ implies $H^{1}(k, \operatorname{Pic}(\bar{C}))=0$.

Moreover, according to Amitsur theorem, the kernel of the restriction map:

$$
r_{X}: \operatorname{Br}(k) \longrightarrow \operatorname{Br}(k(C))
$$

is the cyclic group generated by the class of $[C]$ in $\operatorname{Br}(k)$. Hence, exact sequence (2) induces the following one:

$$
0 \longrightarrow \mathbb{Z} / 2 \longrightarrow \operatorname{Br}(k) \longrightarrow \operatorname{Br}(k(C))
$$

where $k(C)$ is the functions field of $C$.

Example 1.2 If $k$ is a $p$-adic field, $C$ as in example 1.1 then $\operatorname{Br}(k)=Q / Z$, and by Lichtenbaum's duality $\operatorname{Br}(C) \simeq Q / Z \simeq \widehat{\operatorname{Pic}(C)}$, so that (2) becomes :

$$
0 \longrightarrow \mathbb{Z} / 2 \longrightarrow Q / Z \stackrel{\times 2}{\longrightarrow} Q / Z \rightarrow 0
$$

(every class in $H^{2}(C, \mathcal{L})$, where $\mathcal{L}$ is a $C$-lien locally ${ }^{1}$ represented by a semisimple simply connected $C$-group is neutral, cf proof of theorem 2.1 later).

Theorem 1.3 Let $k$ be a local field or a number field or more generally a field of cohomological dimension $\leqslant 2, X$ a proper, smooth rational and geometrically integral $k$-variety. Then $H^{1}(k, \operatorname{Pic}(\bar{X}))$ is finite ${ }^{2}$ and can be inserted in the following exact sequence:

$$
\operatorname{Br}(k) \longrightarrow \operatorname{Br}_{1}(X) \longrightarrow H^{1}(k, \operatorname{Pic}(\bar{X})) \longrightarrow 0
$$

where $\operatorname{Br}_{1}(X)=\operatorname{Ker}\{\operatorname{Br}(X) \longrightarrow \operatorname{Br}(\bar{X})$.

Remark 1 Under Theor.1.3 conditions, if the elementary obstruction ob $(X)$ of $X$ defined in [2] (Def. 2.2.1 page 413) vanishes, i.e the exact sequence:

$$
1 \longrightarrow \bar{k}^{*} \stackrel{\sigma}{\longrightarrow} \bar{k}(X)^{*} \longrightarrow \bar{k}(X)^{*} / \bar{k}^{*} \longrightarrow 1
$$

$\overline{1 \text { for etale topology }}{ }^{2}$ cf [2] Corollary 2.A.2, p.461. 
splits, i.e there exists a retraction $\sigma: \bar{k}(X)^{*} \longrightarrow \bar{k}^{*}$, then $(3)$ can be inserted in the following diagram:

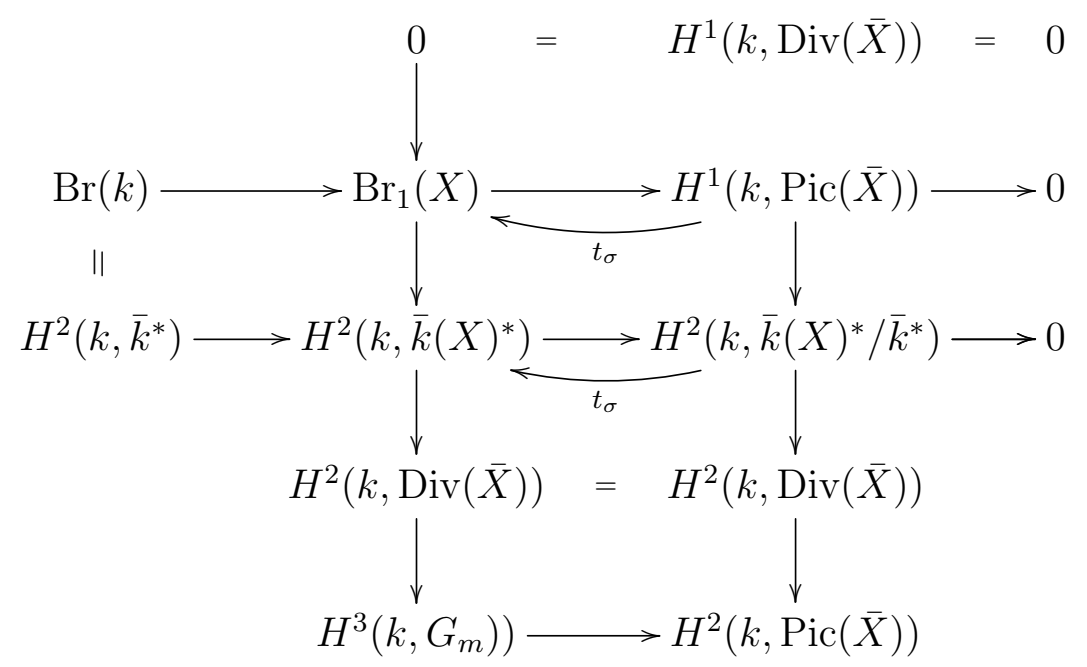

where $t_{\sigma}$ is induced by section $\sigma$.

Therefore $o b(X)=0$ implies, additionally, the existence of a retraction $t_{\sigma}$ in the exact sequence (3):

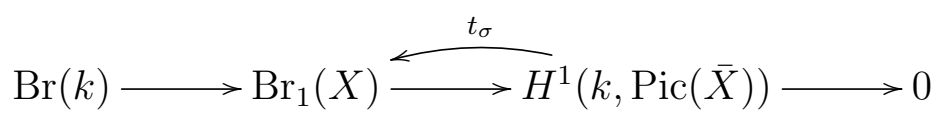

and $\operatorname{Br}_{1}(X)=\operatorname{Br}(X) \oplus H^{1}(k, \operatorname{Pic}(\bar{X}))$.

The group $H^{1}(k, \operatorname{Pic}(\bar{X}))$ is then closely related to the Brauer group of $X$ :

- If $X$ is $k$-rational, $\operatorname{Pic}(\bar{X})$ is a stable permutation $\bar{k} / k$-module, hence $H^{1}(k, \operatorname{Pic}(\bar{X}))=0$.

- If $H^{1}(k, \operatorname{Pic}(\bar{X})) \neq 0$, we obtain on an obstruction to $k$-rationality.

\section{Reduction of the cohomology of $X$}

In this $\S, X$ is as in $\S 1$ : a proper, smooth, geometrically integral and rational $k$-variety.

We consider cohomology with values in a lien $\mathcal{L}$ locally ${ }^{1}$ represented by a semi-simple simply connected $X$-group $\widetilde{G}$, which we reduce to the cohomology of $X$-tori and the Brauer group of an etale covering of $X$.

The lien $\mathcal{L}$ is an element of $Z^{1}(X$, Autext $\widetilde{G})$. We know by [5], Chap. V, $\S 3$, p. 74 , that the exact sequence :

$$
1 \longrightarrow \widetilde{G}_{a d} \longrightarrow \text { Aut } \widetilde{G} \longrightarrow \text { Autext } \widetilde{G} \longrightarrow 1
$$


splits and therefore $\mathcal{L}$ defines a class $\left[\widetilde{G}_{\mathcal{L}}\right]$ in $H^{1}(X$, Aut $\widetilde{G}): \mathcal{L}$ is represented by $\operatorname{par} \widetilde{G}_{\mathcal{L}}$.

$\widetilde{G}_{\mathcal{L}}$ is semi-simple simply connected quasi-split: it admits a Killig pairing $(\widetilde{B}, \widetilde{T})$ where $\widetilde{T}=\prod_{X^{\prime} / X} G_{m_{X^{\prime}}}$ is an induced torus of the finite etale covering $X^{\prime}$ of $X$, where $X^{\prime}=X \underset{k}{\otimes} k^{\prime}, k^{\prime}=\operatorname{Dyn}\left(\widetilde{G}_{\mathcal{L} / k}\right)$ refers to the Dynkin $k$-scheme of $\widetilde{G}_{\mathcal{L}}$ (cf. Prop. 3.13-exposé XXIV of [9]). We have then the diagram:

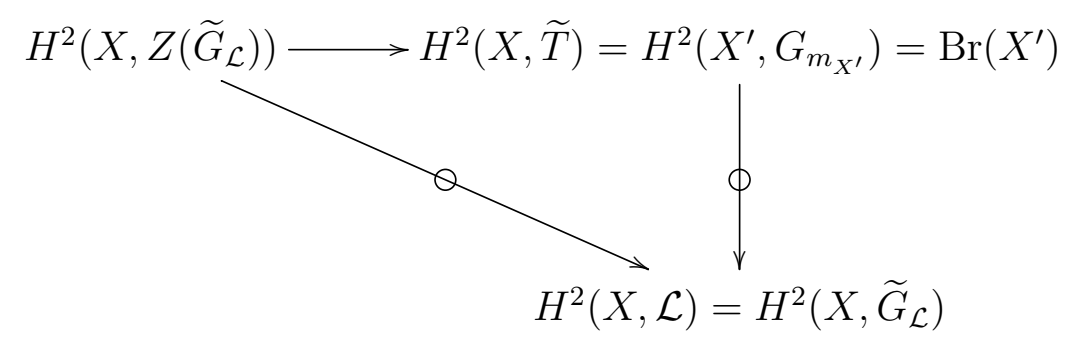

where $\stackrel{0}{\longrightarrow}$ is the Giraud's relation [8].

$H^{2}\left(X, \widetilde{G}_{\mathcal{L}}\right)$ is a principal homogeneous space under $H^{2}\left(X, Z\left(\widetilde{G}_{\mathcal{L}}\right)\right)$ : let $q \in H^{2}\left(X, \widetilde{G}_{\mathcal{L}}\right), q$ can be written as $q=\alpha \cdot \varepsilon$ where $\varepsilon=\left[\right.$ Tors $\left.\widetilde{G}_{\mathcal{L}}\right]$ and $\alpha \in H^{2}\left(X, Z\left(\widetilde{G}_{\mathcal{L}}\right)\right)$. The class $q$ is then related to the image of $\alpha$ in $H^{2}(X, \widetilde{T})=$ $\operatorname{Br}\left(X^{\prime}\right)$.

Theorem 2.1 Let $k$ be a p-adic field, or a number field, or a field of characteristic 0 , of cohomological dimension $\leqslant 2$, such that for every finite simple central $k$-extension algebra, the index equals the exponent. If $X$ is $k$-rational variety, $\widetilde{G}$ a semi-simple simply connected $X$-group, $\mathcal{L}$ an $X$-lien locally ${ }^{1}$ represented by $\widetilde{G}$, then every class in $H^{2}(X, \mathcal{L})$ is neutral.

Indeed, since $X$ is $k$-rational, $X^{\prime}$ is obviously also $k^{\prime}$-rational, where $k^{\prime}$ refers to the finite extension over which $X^{\prime}$ is defined. Hence $H^{1}\left(k^{\prime}, \operatorname{Pic}\left(\bar{X}^{\prime}\right)\right)=0$ and $\operatorname{Br}\left(k^{\prime}\right)$ maps surjectively on $\operatorname{Br}\left(X^{\prime}\right)$. Diagram $\left(D_{2}\right)$ leads to the following one :

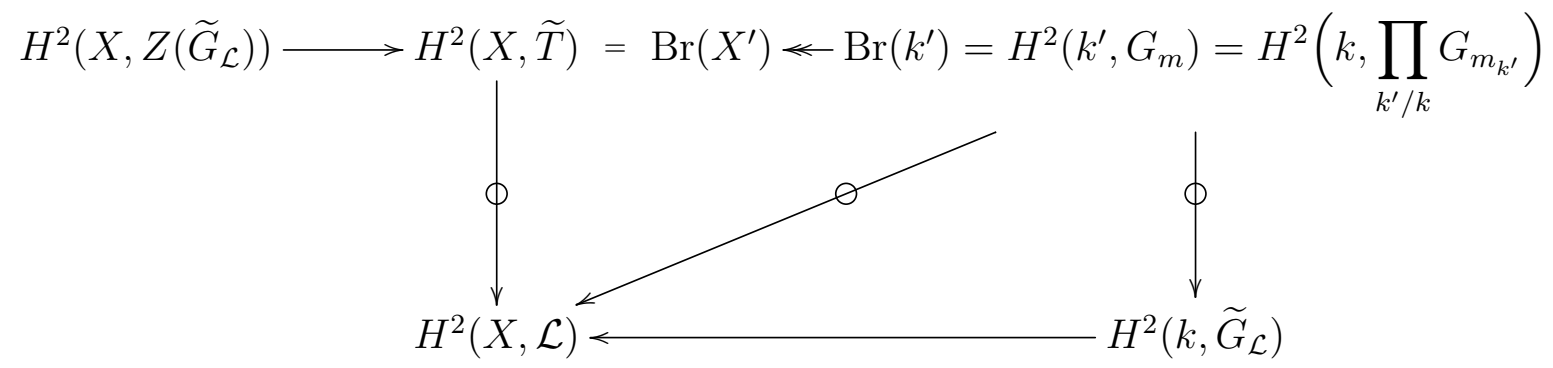

Every class in $H^{2}(X, \mathcal{L})$ can be pulled back to a class in $H^{2}\left(k, \widetilde{G}_{\mathcal{L}}\right)$, which is neutral by [7] or [5] results, and [4] (Prop. 5.3 and following Remark). 


\section{Applications}

1. If $X$ is a proper smooth surface on a number field, that contains a dense open subset which is a $k$-torsor under a torus, then $H^{1}(k, \operatorname{Pic}(\bar{X}))=0$ (cf. Prop. 6.3.2, p. 131 of [10]):

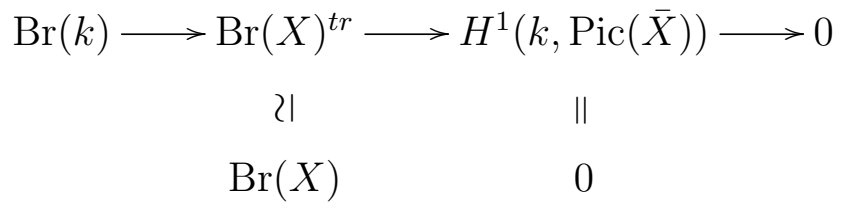

Isomorphism $\operatorname{Br}(X)^{t r} \simeq \operatorname{Br}(X)$ is due to $\operatorname{Br}(\bar{X})=0$, since $\bar{X}$ is $\bar{k}$ rational. $X$ is also $k$-rational once it has a rational point. (here $X$ has a generic $k(X)$-point).

2. Let $k$ be a $p$-adic field or a number field, $X \subset \mathbb{P}_{k}^{n}$ a smooth quadric. For $n \geq 4, \operatorname{Br}(k) \simeq \operatorname{Br}(X)$ (prop.1.6 of [1]). Hence, same conclusion as in Theor 2.1.

Example 2.2 Denote by $X$ the cubic surface $T_{0}^{3}+T_{1}^{3}+T_{2}^{3}+a T_{3}^{3}=0$ defined over a number field $k$. We know that $H^{1}(k, \operatorname{Pic}(\bar{X})) \simeq(Z / 3 Z)^{2}$.

Theorem 2.3 Let $k$ be a number field, $X / k$ a cubic surface as above, $\widetilde{G}$ a split semi-simple simply connected $X$-group and $\mathcal{L}=\operatorname{lien}(\widetilde{G})$. If $|Z(\widetilde{G})|$ is prime to 3 , then every class in $H^{2}(X, \mathcal{L})$ can be pulled back to a class in $H^{2}(k, \mathcal{L})$ and hence, neutral by par [5].

Same result if $k=\mathbb{Q}$ and if $X$ is a Tsfasman $\mathbb{Q}$-surface and $|Z(\widetilde{G})|$ odd; for such surface we know indeed, that $H^{1}(\mathbb{Q}, \operatorname{Pic}(\bar{X}))=Z / 2$.

\section{Main result}

Assume that $X$ is a proper, smooth, geometrically integral $k$-variety which is $\bar{k}$-rational and for which the elementary obstruction [2] vanishes. We know that vanishing elementary obstruction is equivalent to the existence of a universal $X$-torsor. ( $c f$. prop. 2 of [2']). $X$ admits then a universal $X$-torsor $\mathcal{T}$. Let $\mathcal{T}_{c}$ be a smooth $k$-compactification of $\mathcal{T}$. We know that $\mathcal{T}_{c}$ is a rational $k$-variety for which $\operatorname{Pic}\left(\overline{\mathcal{T}}_{c}\right)$ is a stable permutation $\bar{k} / k$-module, hence $H^{1}\left(k, \operatorname{Pic}\left(\overline{\mathcal{T}}_{c}\right)\right)=0$ (cf. Theor.2.1.2 p.411 of [2], or Theor.1 of [3]). We have then the following 
diagram:

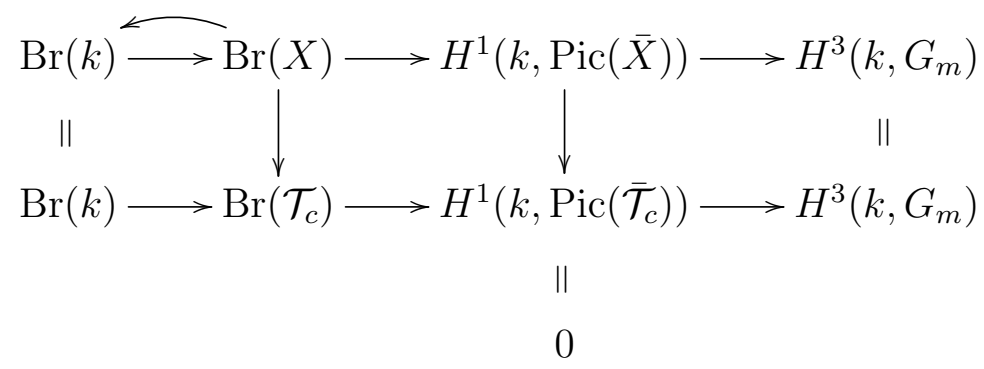

Retraction $\operatorname{Br}(X) \rightarrow \operatorname{Br}(k)$ is due to ob $(X)=0$ condition (cf. large diagram $\left(D_{1}\right)$ of $\S 1$.

Replace in previous diagram $X$ by $X^{\prime}=X \underset{k}{\otimes} k^{\prime}$, where $k^{\prime}=\operatorname{Dyn}\left(\widetilde{G}_{\mathcal{L} / k}\right)$ is the Dynkin $k$-scheme of $\widetilde{G}_{\mathcal{L}}$, we obtain the new diagram :

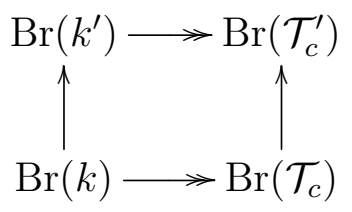

where $\mathcal{T}^{\prime}$ (resp. $\mathcal{T}_{c}^{\prime}$ ) is a universal $X^{\prime}$-torsor, restriction to $X^{\prime}$ of universal $X$-torsor $\mathcal{T}\left(\operatorname{resp} . \mathcal{T}_{c}\right)$.

Next diagram, and especially surjectivity of $\operatorname{Br}\left(k^{\prime}\right) \longrightarrow \operatorname{Br}\left(\mathcal{T}_{c}^{\prime}\right)$ :

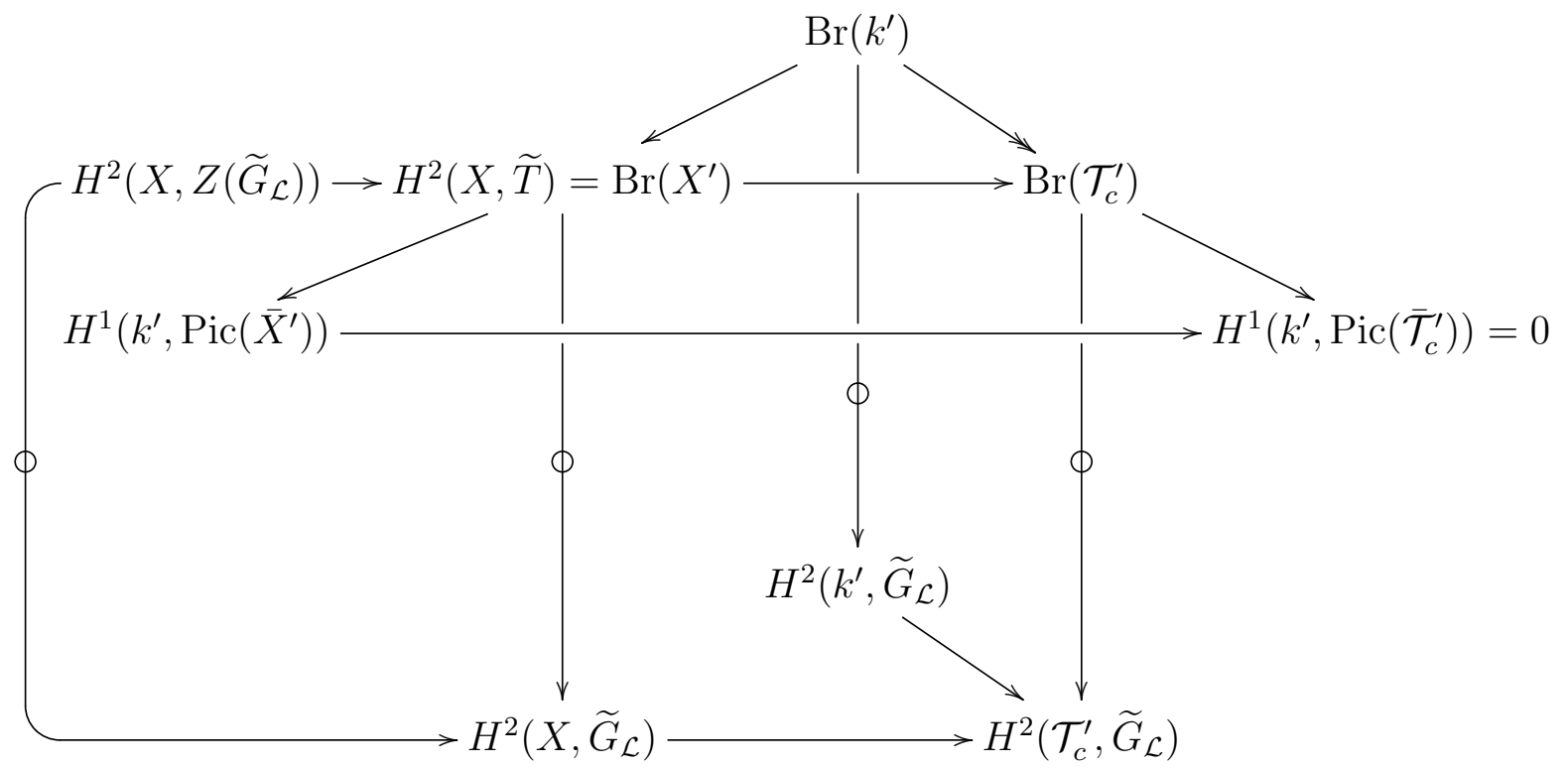

$\left(D_{4}\right)$

show then that every class of $\operatorname{Im}\left(H^{2}\left(X, \widetilde{G}_{\mathcal{L}}\right) \longrightarrow H^{2}\left(\mathcal{T}_{c}^{\prime}, \widetilde{G}_{\mathcal{L}}\right)\right)$ comes from $\operatorname{Im}\left(H^{2}\left(k^{\prime}, \widetilde{G}_{\mathcal{L}}\right) \longrightarrow H^{2}\left(\mathcal{T}_{c}^{\prime}, \widetilde{G}_{\mathcal{L}}\right)\right)$. Hence theorem : 
Theorem 3.1 Let $k$ be a perfect field, $X$ a proper, smooth and geometrically integral $k$-variety which is $\bar{k}$-rational and for which elementary obstruction $o b(X)=0$.

Then to every $X$-lien $\mathcal{L}$ locally ${ }^{1}$ represented by a semi-simple simply connected $X$-group $\widetilde{G}$, there is an associated finite etale covering $X^{\prime}=X \underset{k}{\otimes} k^{\prime}$ of $X$ so that every class in :

$$
\begin{aligned}
& \operatorname{Im}\left(H^{2}(X, \mathcal{L}) \longrightarrow\right.\left.H^{2}\left(X^{\prime}, \mathcal{L}_{X^{\prime}}\right) \longrightarrow H^{2}\left(\mathcal{T}_{c}^{\prime}, \mathcal{L}_{\mathcal{T}_{c}^{\prime}}\right)\right) \\
& \| \| \\
&\left.H^{2}\left(X^{\prime}, \widetilde{G}_{\mathcal{L}}\right)\right) \longrightarrow H^{2}\left(\mathcal{T}_{c}^{\prime}, \widetilde{G}_{\mathcal{L}}\right)
\end{aligned}
$$

is in $\operatorname{Im}\left(H^{2}\left(k^{\prime}, \mathcal{L}\right) \rightarrow H^{2}\left(\mathcal{T}_{c}^{\prime}, \mathcal{L}_{\mathcal{T}_{c}^{\prime}}\right)\right)$.

Corollary 3.2 Let $k$ be a field as in theorem 2.1, $X$ a proper, smooth and geometrically integral $k$-variety which is $\bar{k}$-rational and so that $o b(X)=0$, then to every $X$-lien $\mathcal{L}$ locally ${ }^{1}$ represented by a semi-simple simply connected $X$-group $\widetilde{G}$ is associated a finite etale covering $X^{\prime}=X \underset{k}{\otimes} k^{\prime}$ of $X$ so that every class $H^{2}(X, \mathcal{L})$ is neutralized by the base change from $X$ to $\mathcal{T}_{c}^{\prime}$ induced by $\mathcal{T}_{c}^{\prime} \rightarrow X^{\prime} \rightarrow X$, where $\mathcal{T}_{c}^{\prime}$ is a smooth compactification of a universal $X^{\prime}$ torsor.

Indeed, if $k$ is a number field or a $p$-adic field, then every class in $H^{2}(k, \mathcal{L})$ is neutral. ([5],[6]).

If $k$ is of characteristic 0 , of cohomological dimension $\leqslant 2$ and so that for every simple central algebra on a finite extension of $k$, the index equals the exponennt, $c f$. la prop. 5.3 de [4].

The previous theorem corroborates the fact that universal torsors compactifications have more simpler arithmetic.

Corollary 3.3 Under theorem 4 conditions, every class in $H^{2}(X, \mathcal{L}) / H^{2}(k, \mathcal{L})$ is neutralized on $H^{2}\left(\mathcal{T}_{c}^{\prime}, \mathcal{L}_{\mathcal{T}_{c}^{\prime}}\right) / H^{2}\left(k^{\prime}, \mathcal{L}\right)$.

\section{References}

[1] J-L Colliot-Thélène \& M. Ojanguren, Variétés unirationnelles non rationnelles: au-delà de l'exemple d'Artin et Mumford, Inv. Math. 97 (1989), N1, 141-158.

[2] J-L Colliot-Thélène \& J-J. Sansuc La descente sur les variétés rationnelles II, Duke Math. J. 54 (1987), No. 2, 375-492.

1 for etale topology. 
[2'] J-L Colliot-Thélène \& J-J. Sansuc Variété de première descente attachées aux variétés rationnelles, C.R Acad. Sc. Paris, t. 284 (25 avril 1977), Série A, 967-970.

[3] J-L Colliot-Thélène \& J-J. Sansuc La descente sur les variétés rationnelles, Journées de Géometrie Algébrique d'Angers, Juillet 1979, pp. 223-237.

[4] J.L. Colliot-Thélène, P. Gille \& R. Parimala Arithmetic of linear algebraic groups over 2-dimensional geometric fields, Duke Math. J. 121 (2004), 285-341.

[5] J-C Douai 2-Cohomologie galoisienne des groupes semi-simples, Thèse de doctorat (1976), Université Lille1 - Sciences et Technologies, Editions univerisitaires européennes de Sarebruck, 2010

[6] J-C Douai Sur la 2-cohomologie galoisienne de la composante résiduellement neutre des groupes réductifs connexes définis sur les corps locaux, C. R., Math., Acad. Sci. Paris 342, No. 11, 813-818 (2006)..

[7] J-C Douai 2-Cohomology of semi-simple simply connected group-scheme over curves defined over p-adic fields, Journal de Théorie des Nombres, t. 25, n2 (2013), pp 307-316.

[8] J. Giraud Cohomologie non abélienne, Grundlheren Math. Wiss. vol. 179, Springer-Verlag, 1971.

[9] S.G.A.D., Séminaire de géometrie algébrique du Bois Marie 1963-1964, Lectures Notes in Math., 153 (1970).

[10] A. Skorobogatov Torsors and rational points, Cambrige University Press (2001). 